\title{
Intermédialités
}

Histoire et théorie des arts, des lettres et des techniques

Intermediality

History and Theory of the Arts, Literature and Technologies

\section{Présentation : aimer - la technique}

\section{Éric Méchoulan}

Numéro 4, automne 2004

Aimer

Loving

URI : https://id.erudit.org/iderudit/1005474ar

DOI : https://doi.org/10.7202/1005474ar

Aller au sommaire du numéro

Éditeur(s)

Centre de recherche sur l'intermédialité

ISSN

1705-8546 (imprimé)

1920-3136 (numérique)

Découvrir la revue

Citer ce document

Méchoulan, É. (2004). Présentation : aimer - la technique. Intermédialités /

Intermediality, (4), 9-17. https://doi.org/10.7202/1005474ar d'utilisation que vous pouvez consulter en ligne.

https://apropos.erudit.org/fr/usagers/politique-dutilisation/ 


\title{
Présentation : aimer - la technique
}

\author{
Éric Méchoulan
}

M algré ses tours et ses détours, le verbe « aimer » pourrait apparaître comme nous devenus brutalement sentimentaux, prêts à soumettre la pureté de l'investigation scientifique ou l'incontournable matérialité des dispositifs techniques au bâillement indiscret de la littérature à l'eau de rose et des pastorales postmodernes? Si encore nous avions intitulé ce numéro «désirer», au moins aurions-nous pu récupérer les énergies tacites de la psychanalyse et des multiples pensées qui ont mis cette notion au cœur de leurs propos. Mais c'est peutêtre justement ce décalage par rapport aux métamorphoses enchantées du désir qu'il valait la peine de souligner, en même temps que ce léger caractère désuet et naïf du verbe «aimer» dans notre monde si savant, puisqu'il rend obstinément dissemblables les configurations historiques dans lesquelles il prend son sens. Dans une certaine mesure, le désir n'a pas d'histoire, alors que l'amour, qui en mobilise l'énergie dans des figures de séduction, dans des usages de civilité ou dans des rituels de perversion, n'existe que dans et par l'histoire. C'est reconnaître qu'aimer est toujours une affaire de technique, depuis l'érudition minutieuse des corps dans les grandes manœuvres du sexe (dont la Chine et l'Inde anciennes ont fait de prodigieux traités) jusqu'aux mises en scène illusoires de l'amour-propre ou vertueuses de la charité, en passant par l'amour des signes dont la courtoisie médiévale, la galanterie classique, le roman réaliste et le cinéma contemporain, chacun à leur manière, ont fait un véritable médium de communication. Il faut, en effet, le souligner : aimer n'a rien de naturel, c'est une invention des hommes. 


\section{Le MYTHE DE LA TECHNIQUe}

Afin de mieux appréhender comment la technique se glisse facilement sous les apprêts enchanteurs de l'amour, reprenons brièvement un mythe ancien - un mythe d'origine, comme tout mythe. C'est un sophiste qui parle sous l'allègre écriture de Platon dans un dialogue qui commence par une évocation des amours de Socrate:

C'était le temps où les dieux existaient déjà, mais où les races mortelles n'existaient pas encore. Quand vint le moment marqué par le destin pour la naissance de celles-ci, voici que les dieux les façonnent à l'intérieur de la terre avec un mélange de terre et de feu et des substances qui se peuvent combiner avec le feu et la terre. Au moment de les produire à la lumière, les dieux ordonnèrent à Prométhée et à Épiméthée de distribuer convenablement entre elles toutes les qualités dont elles avaient été pourvues. Épiméthée demanda à Prométhée de lui laisser le soin de faire lui-même la distribution. [...] Or Épiméthée, dont la sagesse était imparfaite, avait déjà dépensé, sans y prendre garde, en faveur des animaux [aloga], toutes les facultés [dunameis] dont il disposait et il lui restait encore à pourvoir l'espèce humaine pour laquelle, faute d'équipement, il ne savait que faire. Dans cet embarras, survient Prométhée pour inspecter le travail. Celui-ci voit toutes les autres races [ta zôa, les animaux] harmonieusement équipées et l'homme nu, sans chaussures, sans couvertures, sans armes. Et le jour marqué par le destin était venu, où il fallait que l'homme sortît de la terre pour paraître à la lumière. Prométhée, devant cette difficulté, ne sachant quel moyen de salut trouver pour l'homme se décide à dérober l'habileté artiste [tèn entechnon sophian] d'Héphæstos et d'Athéna et, en même temps, le feu - car, sans le feu, il était impossible que cette habileté fût acquise par personne ou rendît aucun service - , puis, cela fait, il en fit présent à l'homme. C'est ainsi que l'homme fut mis en possession des arts utiles à la vie [peri ton bion sophian $]^{1}$.

La fable très connue du vol de Prométhée trouve donc son ancrage dans ce mythe: à la différence des animaux, tous bien pourvus de ce qui est nécessaire à leur existence, l'homme naît sans rien, et le savoir technique que Prométhée dérobe aux dieux assure seul sa conservation. Les animaux vivent grâce aux qualités qui leur ont été allouées; les hommes survivent simplement par l'habileté technique. Le manque originel et le vol de Prométhée amènent en effet les hommes à reprendre à leur compte une connaissance des arts et du feu

1. Platon, Protagoras, trad. Alfred Croiset, introduction et notes Pierre-Marie Morel, Paris, Éditions Les Belles Lettres, 1997, p. 37-39, 320 c 7 - $321 \mathrm{~d} 4$. 
que seuls les dieux possédaient: la survie laborieuse des humains devient ainsi une forme de sur-vie, de gain primordial sur l'existence ordinaire des bêtes. Puisqu'il n'a pas reçu originellement les moyens indispensables à sa conservation, l'homme est un être qui vit nécessairement au-dessus de ses moyens. C'est bien cela que l'on nomme technique.

On peut remarquer l'effet d'insistance de Protagoras : tèn entechnon sophian. Il ne s'agit pas simplement d'un savoir technique qui est ainsi volé par Prométhée, mais d'une habileté générale aux techniques, un savoir de l'heureuse technique, peut-être même une sagesse technique (sophia est savoir, science, habileté, intelligence autant que sagesse). C'est cette science des bons moyens qui permet aux hommes de vivre par-delà leurs manques de moyens.

Du coup, le dénuement ou l'inachèvement de l'espèce humaine lui permet de partager de manière imprévue un peu de l'univers des dieux. En profitant de la technique volée à Athéna, les hommes reconnaissent qu'il existe des êtres supérieurs et leur expriment alors leur reconnaissance sous formes de rites religieux. Par «savoir technique», il faut en effet nous extirper de la seule dimension technologique de fabrication d'outils et d'objets nécessaires à la vie. Pour Protagoras, le premier élément qui découle du vol fait aux dieux, ce sont justement les techniques des rituels religieux; le second tient à la voix et aux mots qui font cette fois la technique du langage (les animaux ne sont pas désignés seulement par le terme habituel ta zôa, mais aussi par ta aloga, les sans parole ou les sans raison) - autrement dit, il ne faut surtout pas opposer technè à logos, c'est l'heureuse sagesse technique qui ouvre à la race des hommes toute la dimension du langage et de la raison. Les outillages fabriqués et les produits cultivés ne viennent qu'après les honneurs rendus aux dieux et les discours échangés entre les hommes.

\section{Aparté néoténique}

Il est amusant de voir que la science contemporaine redécouvre, dans les origines de l'homme, l'immaturité mythique et l'ouverture à la technique dont parle Protagoras. Il existe, en effet, une espèce animale qui fascine les zoologues depuis plusieurs décennies: les axolotls. Ce sont des formes larvaires d'un batracien des plus communs du genre amblystone. Si on leur injecte certaines hormones thyrö̈diennes, la mue se produit et ils changent leur délicate peau rose contre l'aspect plutôt révulsant de salamandres marbrées - mais il faut cette intervention humaine pour les faire échapper à leur état larvaire, comme s'ils étaient les échantillons rares d'un blocage infantile. Les scientifiques ont 
longtemps conçu cette fixation larvaire (respiration branchiale, peau d'un rose opalescent, mode de vie exclusivement aquatique) comme une régression dans le destin animal, explicable par certaines conditions d'existence (carence en iode des lacs des hauts plateaux mexicains dans lesquels vivent les axolotls, basses températures, altitude élevée). Mais tout se passe comme si leurs petites têtes triangulaires, mystérieusement aztèques, et leurs corps d'un rose obstiné se refusaient à devenir adultes. Sans donner à ce «refus » la teneur d'une volonté, cet arrêt dans l'évolution normale d'un banal batracien apparaît, pour les spécialistes, comme le résultat d'une inhibition endocrinienne (autrement dit, du système qui contrôle la production thyrö̈dienne, responsable de la mue).

Cependant, un tel blocage au stade larvaire ne suffit pas à constituer les axolotls en espèce animale, car il devrait avoir signé depuis longtemps leur disparition dans la lutte pour l'existence. Le plus fascinant de l'affaire ne tient pas simplement à la fixation à une phase infantile de leur développement, mais à leur capacité acquise de se reproduire. À la différence de toutes les espèces qui n'octroient le pouvoir de reproduction qu'aux adultes, les axolotls sont des bébés pubères.

Pareille bizarrerie de l'évolution animale jette un certain éclairage sur la philogenèse de l'homo sapiens. On a remarqué bien souvent, dans la dérivation entre primates et humains, que ceux-ci ressemblaient beaucoup moins aux adultes qu'aux bébés primates (même relative absence de pilosité, même position du trou occipital, même structure des mains et des pieds, même pli mongolien, etc.). Il serait alors possible de concevoir que la véritable spécificité des humains, dans l'évolution des espèces animales, vient de ce qu'ils sont des primates fixés à un stade infantile, mais ayant conquis la faculté de se reproduire. Le stade transitoire de l'enfance pour un primate serait devenu, pour l'homme, un état définitif. Telle est ce que l'on nomme «l'hypothèse de la fœtalisation » qui trouve l'essence de l'homme dans le caractère retardé de son développement ${ }^{2}$.

2. Un des premiers à proposer cette idée de l'homme comme espèce retardée a été l'anatomiste hollandais Louis Bolk dès les années 1920-1930 (voir Louis Bolk, «La genèse de l'homme", Arguments, no 18, 1960, p. 3-13). Arnold Gehlen a tâché de suivre les implications de ce qu'il appelle un «infantilisme normalisé » dans une théorie anthropologique plus générale, dès les années 1950; voir Arnold Gehlen, Anthropologie et psychologie sociale, trad. Jean-Louis Bandet, Paris, Presses Universitaires de France, 1990 [1986]. 
On ne peut certes faire des expériences comme avec les axolotls et injecter à des êtres humains des hormones thyroïdiennes afin de voir s'ils deviennent effectivement des primates patentés, mais certaines maladies endocriniennes montrent que l'inhibition de la thyrö̈de qui gère ces métamorphoses physiologiques peut être ponctuellement levée et provoquer, de fait, un accroissement de la pilosité, des développements de l'os frontal et de la mâchoire, une puberté inhabituellement précoce: modifications qui nous paraissent pathologiques, mais que l'on pourrait prendre au contraire pour l'actualisation de notre destin animal.

En un sens, si la sexualité dépasse chez l'homme la simple reproduction de l'espèce et si nous l'entourons de tant de prestigieux plaisirs, c'est que, fondamentalement, à l'instar de la technique volée par Prométhée, nous n’y avons pas droit: d'avoir usurpé le pouvoir de reproduction réservé aux adultes nous laisse, du coup, libres d'en user à tort et à travers. Sexualité et liberté vont de pair dans le monde des hommes. S’il n'en va pas de la sorte pour les organismes néoténiques des axolotls - la «néoténie » est le nom donné par les biologistes pour désigner cette fixation fotale - , c'est que les hommes apparaissent sur-retardés. Nous naissons et demeurons inachevés (il nous faut des années pour apprendre à marcher, à nous nourrir, à développer des souvenirs), en retard sur toutes les autres espèces animales ${ }^{3}$ - et c'est justement ce que nous appelons être plus évolués!

Chez les animaux que nous nous plaisons à considérer comme les moins évolués (les insectes par exemple), le processus moteur est rigoureusement déterminé : pour se nourrir, telle espèce de guêpe solitaire pique inéluctablement le ganglion ventral de son unique proie, la larve du scarabée doré. La précision géométrique du geste n’est pas le résultat d’un apprentissage, mais une formation biologique inscrite dans la structure génétique de l'insecte comme un destin écrit par les dieux. Notre enfance prolongée nous a, au moins en partie, libérés d'une telle structuration, elle nous a plongés dans une plasticité indéfinie ${ }^{4}$ - et c'est bien pour limiter et contrôler cette effrayante souplesse

3. Voir Geza Roheim, The Origin and Function of Culture, New York, Nervous and Mental Diseases Monographs, 1943.

4. Plusieurs philosophes ont tenté d'en tirer des réflexions sur notre actualité même: Giorgio Agamben, Idée de la prose, Paris, Éditions Christian Bourgois, 1988 [1985]; DanyRobert Dufour, Lettres sur la nature humaine à l'usage des survivants, Paris, Éditions Calmann-Lévy, 1999; Peter Sloterdijk, Règles pour le parc humain: une lettre en réponse à la Lettre sur l'humanisme de Heidegger, trad. Olivier Mannoni, Paris, Éditions Mille et une nuits, 2000 [1999]. 
d'âme que nous avons imaginé de multiples façons de nous contraindre (à commencer par l'invention même de l'«âme» ou des interdits sexuels).

Une de ces contraintes est le langage. Il est courant de penser l'homme comme l'animal doté de la parole. Rien n'est moins évident. Les animaux naissent dans le langage, alors que les humains sont ces animaux sans langage qui doivent justement l'acquérir, donc l'inventer. Du coup, le langage change de sens: il n'offre plus une discipline des comportements, liant de façon répétitive et nécessaire tel signal à telle action; il ouvre au vertige des nominations, aux beautés des synonymies, au labyrinthe des règles et des catégories; il disjoint et accouple la singularité d'un événement et la généralité d'une signification. Dans l'animal, l'empirique et le transcendantal sont impeccablement confondus; l'être humain œuvre, au contraire, sur la difficile jointure des deux.

L'animal, parce qu'il naît dans un code, ne le saisit jamais comme code; il en réitère obstinément les effets. L'être humain, puisqu'il doit rendre possible une communication, donc une communauté, est à l'origine de ses langues: il ne les domine pas individuellement, mais il en maîtrise les possibilités. Il ne les domine pas, dans la mesure où il est bien doté de la parole, mais non sous la forme d'une inscription génétique, d'une qualité intrinsèque, il lui faut recourir à l'apprentissage: il n'est pas de langage solitaire, et jamais un homme n'en a été pourvu autrement que par la tradition et l'enseignement d'autres humains. L'être humain est donc bien, lui aussi, dans le langage dès sa naissance, mais suivant un ordre tout différent de celui des animaux: là où ceux-ci le reçoivent selon un processus quasi immuable de génération en génération, celui-là en hérite et en fait son propre bien selon des processus toujours neufs, ouverts à la fois sur les possibles et les nécessités, pris dans des dettes et des obligations. En figurant comme l'inachevé, le retardé au compte courant des espèces, l'être humain devient un animal historique: l'histoire naît avec la nécessité de l'héritage. Il faut porter à son crédit ce dérimage des temps qui fait d'un retard un progrès.

Ce à quoi les animaux sont aveugles n'est jamais le signe. Le monde, pour eux, fourmille, au contraire, de signes. Mais c'est l'opacité du signe lui-même qu'ils ne peuvent appréhender. Le retard du développement et l'apprentissage du langage font que les signes apparaissent enfin, existent aussi par eux-mêmes. La «pensée » est issue de cette réflexion inaugurée par la réflexivité des signes, c'est-à-dire de leur double capacité à s'abolir dans la dénotation d'un référent et à se manifester dans leur vertu de signe, à s'effacer dans une transparence insoupçonnée et à se montrer selon la sensualité de l'opaque. De tous les animaux, l'être humain est celui qui, régulièrement, lâche la proie pour l'ombre. 


\section{De la politique comme technique d’Aimer}

Cependant, comment empêcher que les êtres humains ne deviennent les proies très matérielles d'autres humains. Comment faire pour qu'un ordre commun puisse accorder ces animaux qui ont raté leur destin animal et dont la liberté se voit rapidement bloquée par les désirs des autres humains? Revenons alors au mythe de Protagoras et, plus directement, à la question de la technique et de son enjeu proprement politique. Car si le point de départ de la discussion avec Socrate est le fait de savoir ce qu'enseigne exactement Protagoras et quelle en est l'utilité pour un jeune homme, le dialogue consiste surtout à comprendre comment (puisque Protagoras déclare enseigner l'art du parfait citoyen) il serait possible d'enseigner la politique, alors qu'elle est d'office le lot de chacun et que personne ne pouvant se prétendre plus compétent qu'un autre en cette matière (à la différence des métiers qui s'apprennent et instaurent des compétences spécifiques), il ne peut être question de l'enseigner. Le mythe que raconte Protagoras est censé fournir une réponse à ces questions de Socrate. La première partie du mythe consiste donc à montrer en l'homme une immaturité originaire et un supplément de moyens octroyé par le vol du savoir technique par Prométhée.

Mais cela ne suffit pas. Le partage des rituels, des mots, des outils et des produits ne permet pas encore d'édifier une cité où tous les membres demeurent harmonieusement unis. Il manque toujours une manière de lier les humains entre eux, afin qu'ils puissent résister aux bêtes sauvages. Ils se réunissent souvent, bâtissent même des villes, mais aussitôt qu'ils les ont construites, chacun y maltraite et lèse (adikê̂n, 322 b 8) les autres: ils ne savent pas faire d'une ville qui rassemble des individus, une cité (polis) qui ordonne une multitude. C'est donc l'art du politique qui leur fait cruellement défaut. Prométhée n’a pas pu voler la politique en même temps que le feu et les savoirs de la technique, car elle se trouvait dans la demeure même de Zeus, soigneusement gardée. Au plus proche du roi des dieux réside donc l'art de tisser les liens entre les êtres. Pourtant, de même qu'il ne faut pas opposer technè à logos, on ne doit pas trouver dans la politique une envergure contraire à la technique, car depuis le début du dialogue Protagoras a été qualifié de savant en politikèn téchnen (319 a 4) et c'est bien la technique politique dont manquent encore les êtres humains. L'art de vivre ensemble est une forme de technique, différente de la fabrication d'outils et même de la production de discours, car elle requiert une qualité qui est absente de tous en ce que chacun s'avère immédiatement adikos (injuste). 
L'être humain peut conserver sa vie; il ne sait pas encore comment la vivre en commun avec les autres humains. Pour Protagoras, l'invention de la communauté politique passe donc par la justice (dikè) et le respect (aidôs peut aussi vouloir dire «pudeur», voire «révérence») que Zeus se décide à transmettre aux hommes par le petit dieu de la communication, Hermès ${ }^{5}$. Et la distribution est parfaitement égale: chacun peut être plus ou moins doué pour tel ou tel métier, mais l'art du politique est nécessairement le lot de chacun, «afin qu'il y eût dans les villes de l'harmonie [poleon cosmoi] et des liens créateurs d'amitié [desmoi philias sunagogoí]» (322 c 2-3). Le lien ainsi créé entre les hommes est une manière d'aimer (phileîn) sous le signe de la justice et du respect, comme le dialogue lui-même s'écrit sous les auspices de l'amour que porte Socrate à la beauté d'Alcibiade ou à la sagesse sans égale de Protagoras (309 c 11). Il faut donc aux humains immatures, d'abord, la technique des outils et du langage afin de conserver leur vie et, ensuite, la technique politique d'aimer les autres hommes afin de survivre ensemble.

On dira que c'est bien là un programme de sophiste: non seulement le langage y est ramené à une simple technologie, mais l'amour (qui élève tant les hommes au-dessus de leur contingence) n'y est présenté que dans son envergure sociale et techniciste. De même que les usages de la sexualité ne se ramènent pas à une programmation pornographique, les valeurs du fait d'aimer ne devraient pas se réduire à des accointances techniques. Mais cette inscription de l'amour et de la vertu dans l'orbe de la technique politique n'est pas le fait de Protagoras, c'est le sage Socrate qui la provoque! En glissant déjà, sans y insister explicitement, de la question de la technique à celle de la vertu politique, comme s'il s'agissait d'équivalents, Socrate inscrit celle-ci à l'horizon de celle-là. En échangeant finalement de position avec Protagoras, à la fin du dialogue, et en revendiquant la possibilité, voire la nécessité, d'un apprentissage de la vertu politique, il trouve bien dans cette excellence recherchée la vertu d'une technique.

Ce qui permet donc l'harmonie des liens dans la cité tient à cette technique amicale. Je rappelle les termes qui sont ainsi assemblés par SocrateProtagoras: poleon cosmoi. Le cosmos désigne le bel ordre, l'arrangement selon les convenances, l'arrangement au sens de parure, mais aussi le monde des humains et même l'univers en son entier. C'est en faisant, par les liens politiques de

5. Je reviendrai sur ce problème dans le prochain numéro d'Intermédialités consacré justement à cette question de la transmission. 
l'amitié, un cosmos de leurs cités que les êtres humains s'inventent enfin un monde. Les animaux n'ont qu'un milieu de vie (une niche biologique), la néoténie des humains fait aussi qu'ils ont un monde pour y vivre. La technique (en particulier la technique d'aimer) les ouvre à un point de vue qu'il faut bien appeler «cosmopolitique».

Comprend-on mieux maintenant l'intérêt, pour une revue comme Intermédialités, à prendre en compte le fait d'aimer? Loin de tout sentimentalisme verbeux ou de toute pathétique amoureuse, ce sont à des dispositifs techniques (au sens large que je viens d'indiquer), que sont dévolues les études qui suivent, en même temps qu'à l'envergure politique du fait d'aimer. En ce sens, la philologie, qui témoignait d'abord littéralement d'un amour de la langue, trouve son juste prolongement dans l'intermédialité comme amour des moyens: il est donc tout à fait normal que les techniques d'aimer en deviennent des objets prioritaires - avec l'avantage de ne pas s'illusionner trop fortement sur l'impeccable jointure de la dikè et de l'aidôs: le dialogue de Platon lui-même multiplie les occasions de dispute, de conflit, de mésententes, de dissensions, de méprises, voire d'ironiques mépris. L’intermédialité suit les méandres de l'aveugle amour en se mettant à l'écoute de toutes ses techniques. 